\title{
A yaw angle testing system design based on magnetoresistive sensor and accelerometer

\author{
Ping Xia ${ }^{1, a}$, Yangkui Xiang ${ }^{1, b}$, Fuyong Cheng ${ }^{1, c}$ \\ ${ }^{1}$ Unit 63981 of PLA, Wuhan, China 430311
} \\ aXia1987@126.com, b969978706@qq.com, ‘598613258@qq.com
}

Keywords: navigation; yaw angle; magnetoresistive sensor; accelerometer

\begin{abstract}
With the development of aerospace business and the popularization of automobile and yacht, navigation has been an important part of people's life. And in the area of navigation, obtaining of yaw angle appears especially important. This paper designed a electronic compass with angle compensation used magnetoresistive sensor and accelerometer, which has certain reference value to yaw angle calculation of navigation system.
\end{abstract}

\section{Introduction}

Geomagnetic field is one of the basic physical field of the earth, and it is also a vector field. The magnetic field strength and direction corresponding to different location of the area are also different. So, as earth's natural resources, geomagnetic field can be applied to position orientation and attitude control of aircraft or ships and other vehicles. As the improvement of geomagnetic field model and the rapid development of microelectronics and computer technology, geomagnetic navigation has aroused more and more attention in recent years. By ways of geomagnetic navigation, this paper used accelerometer to assist magnetoresistive sensor to calculate the yaw angle when tilt was occured, which has certain application value in the field of navigation.

\section{Calculating Principle Of Yaw Angle}

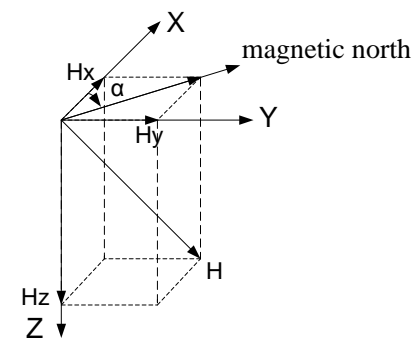

Fig. 1 Definition of azimuth in the XY plane

Fig. 1 shows how to use geomagnetic signal to calculate the yaw angle if tilt isn't in consideration. When pitching and rolling don't exist, the $\mathrm{X}$ axis is forward, $\mathrm{Y}$ axis is horizontal right. The azimuth $\alpha$ is defined as the angle between the direction of the vehicle ( $\mathrm{X}$ axis) and the magnetic North Pole by the clockwise. The angle between $X$ axis and the geographic North Pole is defined as the yaw angle $\Phi$. Then $\Phi=\alpha \pm \beta, \beta$ is the magnetic declination [1,2].

If magnetoresistive sensor measured the geomagnetic signal components $H_{\mathrm{x}}$ and $H_{\mathrm{y}}$, then 


$$
\alpha=\left\{\begin{array}{c}
{[\arctan (H y / H x)] \times 180^{\circ} / \pi,(H x>0, H y>0)} \\
360^{\circ}+[\arctan (H y / H x)] \times 180^{\circ} / \pi,(H x>0, H y<0) \\
180^{\circ}+[\arctan (H y / H x)] \times 180^{\circ} / \pi,(H x<0) \\
90^{\circ},(H x=0, H y>0) \\
270^{\circ},(H x=0, H y<0)
\end{array}\right.
$$

However, in normal condition there is turbulence and tilt to the vehicle's movement inevitably, so we need to re-consider the calculation of the vehicle's yaw angle in $3 \mathrm{~d}$ space when the roll or pitch motion is present.

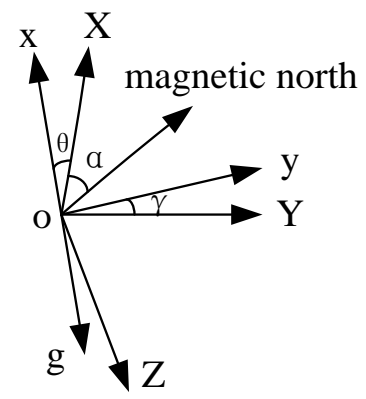

Fig. 2 The real and the ideal coordinate system

As is shown in this coordinate system, the two axis accelerometer can measure gravitation acceleration components Ax and Ay, then

$$
\theta=\arcsin (A x / g) \quad \gamma=\arcsin y(g
$$

We can rotate xoyz coordinate system around $\mathrm{y}$ axis with angle $\theta$, then rotate it around $\mathrm{x}$ axis with angle $\gamma$, thus we can get XOYZ coordinate system (XOY plane overlaps with horizontal plane). So,

$$
\left\{\begin{array}{c}
H_{X}=H x \cos \theta-H z \sin \theta \\
H_{Y}=H x \sin \gamma \sin \theta+H y \cos \gamma+H z \sin \gamma \cos \theta
\end{array}\right.
$$

Put $H y, H x$ into formula (1), then azimuth Angle $\alpha$ can be calculated. The local magnetic declination $\beta$ can refer to the geomagnetic database, then the yaw angle is available.

\section{Hardware design of the system}

Design of geomagnetic signal detection. As acquisition and processing of geomagnetic signal for $1 \mathrm{~d}$ HMC1021Z is the same as the 2d HMC1022, also due to space constraints, only HMC1022 is discussed in this paper. The detection part is based on HMC1022, including the set/reset circuit, sensor circuit, amplifier circuit, filter circuit four parts.

Set/reset circuit. In order to eliminate variation to the output signal and make it the best, the application of SR + / SR -would be required [3]. In chip HMC1022, the set/reset pulse current S/R short to 2 us, at least $4 \mathrm{~A}$ is applied to ensure its high sensitivity and can be read repeatedly. This paper uses the 555 timer circuit to produce the set/reset signal (Fig. 3), and control the P and $\mathrm{N}$ channel HEXFET drives IRF7105 (Fig. 4). The purpose of generating TRS and TSR delay is to ensure that another has been disconnected before a HEXFET connects, which basically is a kind of 
break before make switch cutover mode [4].

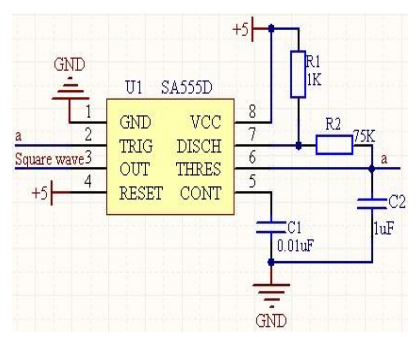

Fig. 3555 timer circuit

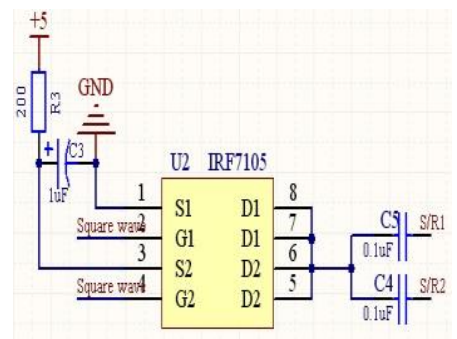

Fig. 4 The S/R pulse generating circuit

Of the 555 timer clock signal, high level time, low level time and clock period is

$$
\begin{aligned}
& t_{H}=0.693(R 1+R 2) C 2 \\
& t_{L}=0.693 R 2 C 2 \\
& T=t_{H}+t_{L}=0.693(R 1+2 R 2) C 2
\end{aligned}
$$

Assume that, $\mathrm{R} 1=1 \mathrm{k}, \mathrm{R} 2=75 \mathrm{k}, \mathrm{C} 2=1 \mathrm{uF}$, then $\mathrm{t}_{\mathrm{H}} \approx 52 \mathrm{~ms}, \mathrm{t}_{\mathrm{L}} \approx 53 \mathrm{~ms}$, duty cycle is about $50 \%[5]$. the signal of IRF7105 is filtered through two $0.1 \mu \mathrm{F}$ capacitor, then S/R1 和 S/R2 connect to the reset pins of HMC1022, thus the pulse can realize reset. At last, Oscilloscope measured that the reset peak current of this design was 4.5A, which meet the requirements of HMC1022 set/reset.

Amplifying circuit. Typical value for geomagnetic field is $0.5 \sim 0.6$ Gauss and sensitivity of HMC1022 is $1 \mathrm{mv} / \mathrm{V} /$ Gauss. With $5 \mathrm{~V}$ power supply, the differential output $\mathrm{V}_{\text {out }}=1 \times(0.5 \sim 0.6) \times 5$ $=(2.5 \sim 3) \mathrm{mv}$. To be convenient for subsequent signal acquisition and processing, signal amplification is needed. Output of the sensor is differential signal at millivolt level. If ordinary amplifier is used directly, then great error will occur because of imbalance voltage of millivolt level and temperature drift of microvolt level. Taking the common mode rejection ratio, temperature drift and gain into consideration, and output signal of magnetoresistive sensor is differential form, then instrumentation amplifier INA128U is used [6]. The amplifier gain can adjust by $\mathrm{R}_{\mathrm{G}} \mathrm{G}=1+50$ $\mathrm{K} \Omega / \mathrm{RG}$.

In this module $\mathrm{G}=1000, \mathrm{R}_{\mathrm{G}}=49.9 \Omega$, enlarged output is $\mathrm{G}\left(\mathrm{U}_{\text {out+ }}-\mathrm{U}_{\text {out- }}\right)$. Amplifier circuit of $A$ direction is shown in Fig. 5.

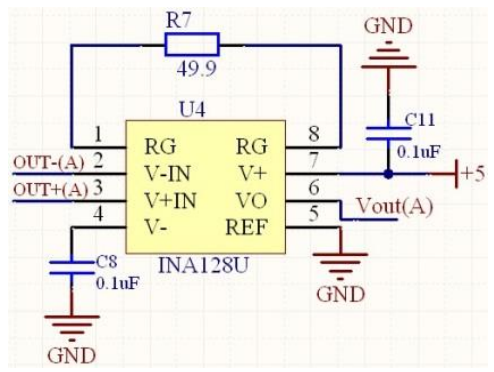

Fig. 5 Amplifying circuit (A direction)

Filter circuit. After signal is amplified but before being sampled and converted, filter processing is still needed. Frequency of geomagnetic signal is very low, which basically is see as a DC signal, so we can choose $100 \mathrm{HZ}$ as the filter cut-off frequency. The filter is designed as second-order low-pass active filter on the basis of LM224 (Fig. 6), and the pass-band cut-off frequency is[7],

$$
\mathrm{fp} \approx 0.37 /(2 \pi \mathrm{RC})
$$


We can order $\mathrm{R}=270 \Omega, \mathrm{C}=2.2 \mu \mathrm{F}$. By testing, it got that the amplitude-frequency characteristic of the filter was flat, and attenuation characteristic was good, which proved that the filter achieved good filtering effect.

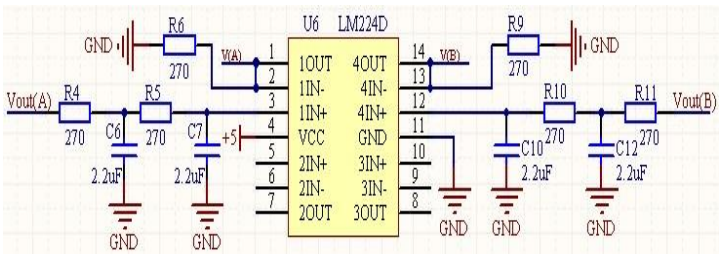

Fig. 6 Active second-order low-pass filter

\section{Software design of the system}

In software design, this system can be divided into two modes as normal mode and calibrating mode. In order to eliminate the biased error caused by imbalance and temperature drift, we need to calculate the average value of the signal when the sensor is under the condition of set and reset, which is called calibrating mode [8]. In normal mode, we need to calculate the yaw angle and compensate the biased error before the output of the attitude angle.

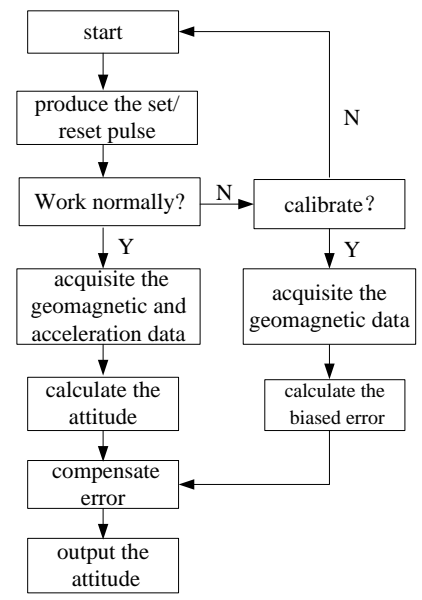

Fig. 7 Software flow chart of the system

\section{The experiment results and analysis}

Finally this system made a real-time display interface of the attitude angle measuring system by python programming language based on the above hardware and software. It showed that the yaw angle error was within $2 \sim 3^{\circ}$ by test repeatedly when tilt occurred. Below pictures are the test results respectively when the system refers to the north and the east under the condition of no tilt, and to the west and the south under the condition of tilt.

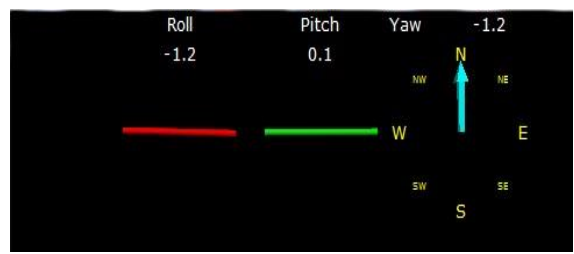

Fig. 8 Refers to the north under no tilt

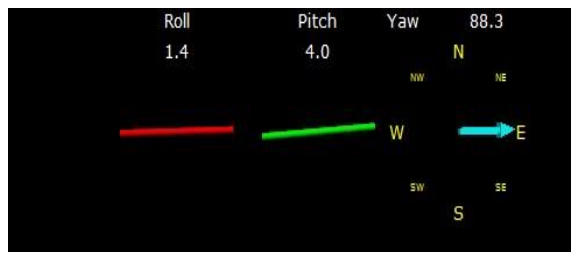

Fig. 9 Refers to the east under no tilt 


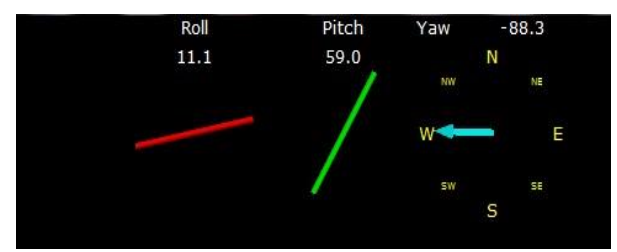

Fig. 10 Refers to the west under tilt

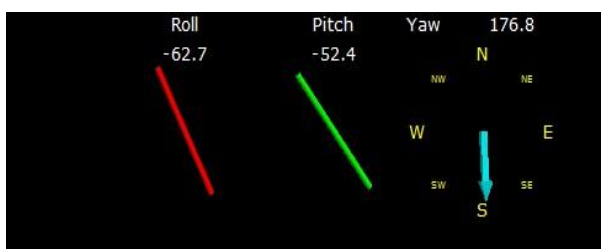

Fig. 11 Refers to the south under tilt

There is a certain error for the calculation of the yaw angle in this system, which comes from all kinds of factors, for the first one is physical error such as manufacturing error and installation error of the sensor, another one is algorithm error such as the pitch and roll error which can lead a further error to the yaw angle. It also needs to further improve at these respects for this system.

\section{References}

[1].Wang Yongqiang. Design of a digital compass based on magnetoresistance sensors[J].Computer Measurement \& Control,2007,(12).

[2].Yan Zhao, Yang Liman. Attitude measurement of driver's head based on accelerometer and magnetoresistive sensor[C]. Proceedings of 2011 International Conference on Fluid Power and Mechatronics, FPM 2011.

[3]. Wang Yonglong, Yang Wei, etc. A weak magnetic signal acquisition system design based on magnetoresistance sensor [J]. Sensors \& Microsystem, 2008, (1).

[4].Li Yida, Zhang Zhongping. Design for 2-axis digital compass with magnetoresistive sensor and microcontroller[J]. Journal of Functional Materials and Devices,2008,(2).

[5].Huang Ping, Pei Suhua. Automation design and application of 555 Timer[J]. Modern Electronic Technology, 2007, (18).

[6]. Wang Jun, Luo Bing. Research of electronic compass with tilt compensation based on the magnetoresistive sensor [J]. Journal of Henan university, 2009, (3).

[7]. Tong Shibai, Hua Chengying. Foundation of analog electronic technology [M]. Beijing: The higher education press, 2006.

[8].Sul Gee Park, Ho Cheol Jeong. Magnetic compass fault detection method for GPS / INS/magnetic compass integrated navigation systems[J].International Journal of Control, Automation, and Systems,2011,(2). 